\title{
Análisis de los factores determinantes de la calidad percibida del servicio prestado por una cooperativa de ahorro y crédito: una aplicación basada en modelos de ecuaciones estructurales
}

\author{
Analysis of the determinants of the perceived quality service provided by credit \\ union: an application based on models of structural equations
}

Hanns de la Fuente Mella ${ }^{1} \quad$ Isabel Díaz Bravo ${ }^{2}$

Recibido 12 de abril de 2011, aceptado 11 de marzo de 2013

Received: April 12, $2011 \quad$ Accepted: March 11, 2013

\begin{abstract}
RESUMEN
En Chile el mercado de instituciones financieras se hace cada día más competitivo, por lo que estas instituciones tienen gran interés por poseer una cartera definida de clientes, es decir, mantener y atraer a sus consumidores asegurando así su rentabilidad y sustentabilidad. Una cartera envejecida puede causar la quiebra de la institución, por lo anterior el esfuerzo debiera centrarse en focalizar gran parte de las acciones hacia el público joven. Esta investigación pretende identificar los factores que determinan la calidad percibida por los clientes jóvenes de una cooperativa de ahorro y crédito, para comprender de qué manera estos producen satisfacción y lealtad hacia una determinada institución financiera. Para ello, la presente investigación plantea una estructura teórica que relaciona diferentes ítems y factores subyacentes, confirmando la misma a través de la metodología de ecuaciones estructurales. Los resultados obtenidos muestran que los componentes más influyentes en la calidad percibida son la imagen corporativa y los aspectos humanos, además, se demuestra que la satisfacción se encuentra en gran medida explicada por la calidad percibida del servicio prestado por la cooperativa de ahorro y crédito analizada. Así, el trabajo pretende añadir valor tanto desde el punto de vista académico como para la gestión de entidades financieras, lo anterior a través de la formulación de una estructura teórica relacional y a través de la identificación de los factores determinantes de la calidad percibida del servicio prestado por una cooperativa de ahorro y crédito.
\end{abstract}

Palabras clave: Calidad percibida, cooperativas, lealtad, satisfacción del cliente, ecuaciones estructurales.

\section{ABSTRACT}

The market of financial institutions becomes increasingly competitive in Chile, therefore these institutions have great interest in having a defined portfolio of clients, which means retain and attract their customers ensuring their profitability and sustainability. An aged portfolio can cause the failure of the institution, so the efforts should focus on targeting many of the actions towards young audiences. The research aims to identifying the factors that determine the quality perceived by young clients of a credit union, to understand how they produce satisfaction and loyalty to a particular financial institution. In order to achieve this goal, this investigation presents a theoretical framework that relates different items and underlying factors, confirming these through the methodology of structural equations. The obtained results show that the most influential components in perceived quality and corporate image are the human factors and also show that satisfaction is largely explained by the perceived quality of service provided by the

1 Pontificia Universidad Católica de Valparaíso. Facultad de Ciencias Económicas y Administrativas. Escuela de Comercio. Avenida Brasil 2830. Valparaíso, Chile. E-mail: hanns.delafuente@ucv.cl

2 Universidad de Talca. Facultad de Ingeniería. Departamento de Modelación y Gestión Industrial. Camino a los Niches km 1. Curicó, Chile. E-mail: isabeldiaz@alumnos.utalca.cl 
credit union analyzed. Thus, the work seeks to add value both from the academic point of view for the management of financial institutions, through the development of a relational theoretical framework and through the identification of the determinants of the perceived quality service provided by a credit union.

Keywords: Perceived quality, credit union, loyalty, customer satisfaction, structural equations.

\section{INTRODUCCIÓN}

Desde tiempos remotos las personas han requerido de financiamiento para sus ideas y proyectos, en respuesta a este requerimiento en el siglo IV a.C. nacen los bancos, los cuales han evolucionado a través de los años. En la actualidad ya no se habla solo de bancos, sino que de un sistema financiero compuesto por: bancos públicos y privados, colectividades locales o regionales, sindicatos, cooperativas, bancos generales y especializados. Cada uno de los cuales atiende a diferentes necesidades de la sociedad actual. Así, las cooperativas son asociaciones autónomas que ayudan a sus asociados a enfrentar sus necesidades económicas, sociales y culturales. Específicamente, las cooperativas de ahorro y crédito tienen como función entregar intermediación financiera en beneficio de sus clientes.

El sistema cooperativo de ahorro y crédito se fundó en Alemania en el siglo XIX, como un sistema de autoayuda, autorresponsabilidad y autoadministración, para el progreso de las familias y empresas. Este sistema ha demostrado una evolución continua y sostenida a través de los años y actualmente es una realidad en los mercados financieros más desarrollados de Europa y Norteamérica, y es una respuesta social en los países donde la banca atiende solamente a una fracción de la población.

Dado el aumento de los niveles de competitividad en el contexto mundial y chileno, las instituciones financieras necesitan reconocer la importancia de atender correctamente las necesidades de sus clientes, con el propósito de alcanzar relaciones a largo plazo con él y lograr mantenerse vigentes en el mercado, es decir, se hace necesario identificar todas las variables que influyen y determinan el comportamiento de los clientes. Así, identificar los factores que afectan a la calidad percibida por los clientes, los elementos que producen su satisfacción y consecuente lealtad hacia una determinada empresa, se hace muy necesario.
La cartera de clientes de las instituciones financieras es uno de sus activos más importantes, ya que esta se relaciona directamente con las utilidades del negocio, así poseer una adecuada cartera de clientes es vital para mantener la vigencia de las entidades financieras.

Una cartera de clientes envejecida pone en riesgo la institución en el largo plazo, por lo tanto, es necesario tomar medidas para renovar dicha cartera $\mathrm{y}$ atraer capitales futuros con el fin de resguardar la existencia de la institución [40].

Particularmente en Chile, según la Superintendencia de Bancos e Instituciones Financieras, el rango etario con mayor número de deudores se presenta entre los 31 y 41 años. Por su parte, la empresa para la cual se desarrollará la investigación posee una edad promedio de clientes de 43 años, es decir, la edad de sus clientes es superior a la de los deudores del sistema financiero, presentando un potencial problema para el futuro.

Una cartera envejecida implica comprometer el futuro y poner fecha de término para la institución; según lo anterior, es necesario identificar los factores atractivos para los clientes jóvenes en el sistema financiero y el grado en que dichos factores conducen a la satisfacción y la lealtad de los clientes hacia la empresa.

Esta investigación pretende identificar los factores que determinan la calidad percibida por los clientes jóvenes de una cooperativa de ahorro y crédito, para comprender de qué manera estos producen satisfacción y lealtad hacia una determinada institución financiera. Por medio de modelos estructurales se estimarán los determinantes de la calidad percibida del servicio financiero, satisfacción y lealtad de los clientes, según lo anterior, se elaborará, aplicará y validará una escala de medida para las variables que afectan el comportamiento de los clientes de una institución financiera, identificando 
y analizando los factores que afectan su satisfacción, con el propósito de presentar una herramienta que oriente las decisiones de la empresa en cuestión.

\section{ESTADO ACTUAL DEL TEMA DE ESTUDIO Y PLANTEAMIENTO DE HIPÓTESIS}

En numerosos estudios diversos autores se han preguntado por cuáles serían los factores y determinantes de una cartera de clientes, la pregunta en cuestión es: ¿captar o mantener a los clientes? Los clientes nuevos son potencialmente más riesgosos que los antiguos, por lo demás, captar un cliente nuevo resulta ser entre cinco y seis veces más costoso que retener a uno antiguo [12, 47]. De este modo se justificaría la importancia de la retención de los clientes dados los beneficios que se generan en las instituciones financieras, por la disminución de los costos operacionales, las referencias, el incremento de las transacciones, la reducción de los costos de cambio, entre otros $[5,24,46]$.

Las actividades orientadas a la retención de los clientes generan una serie de beneficios a las empresas, por lo tanto, es necesario identificar los factores que afectan la satisfacción y lealtad de un cliente con una institución financiera, dado que de este modo se puede conseguir consolidar una cartera de clientes, así como poseer una serie de lineamientos de acción.

Existen numerosos estudios sobre la calidad percibida en las instituciones financieras, cada uno de los cuales expone diferentes dimensiones. Según lo anterior en el Cuadro 1 se muestra un resumen de las principales dimensiones y características consideradas en los diferentes estudios revisados. Estos estudios no se limitan a investigar únicamente la calidad percibida del servicio prestado por instituciones financieras, sino que, además, consideran aspectos como: la imagen corporativa, los aspectos operativos, los aspectos físicos, la tecnología, los aspectos humanos, el servicio recibido y las ventajas de inversión. Debido a lo anterior, este trabajo se centrará en el tema de la calidad percibida, entendida como la habilidad para satisfacer las necesidades de los clientes jóvenes de la cooperativa de ahorro y crédito bajo estudio.

A partir de la revisión de la literatura especializada se propone el modelo de relaciones planteado en la

Cuadro 1. Dimensiones de calidad percibida de servicio en las instituciones financieras.

\begin{tabular}{|c|c|c|c|}
\hline Autor & Dimensiones & Autor & Dimensiones \\
\hline Ishikawa [28] & $\begin{array}{l}\text { Trabajo } \\
\text { Servicio } \\
\text { Información } \\
\text { Proceso } \\
\text { Dirección } \\
\text { Calidad de la empresa }\end{array}$ & Atenas [4] & $\begin{array}{l}\text { Conocer necesidades de los } \\
\text { clientes } \\
\text { Beneficios ofrecidos } \\
\text { Nivel de relaciones } \\
\text { Vías de comunicación, } \\
\text { flujos de información } \\
\text { Tecnología } \\
\text { Lenguaje }\end{array}$ \\
\hline $\begin{array}{l}\text { Newby and } \\
\text { McManus [42] }\end{array}$ & $\begin{array}{l}\text { Calidad global de atención } \\
\text { Escuchar, entender y } \\
\text { responder en forma rápida y } \\
\text { eficaz }\end{array}$ & González [25] & $\begin{array}{l}\text { Definición y medición de las } \\
\text { exigencias de los clientes } \\
\text { Obtención de las } \\
\text { expectativas de los clientes }\end{array}$ \\
\hline $\begin{array}{l}\text { Valdunciel, Flórez } \\
\text { y Dávila [56] }\end{array}$ & $\begin{array}{l}\text { Gama de productos y servicios } \\
\text { Esfuerzos por mantener la } \\
\text { ausencia de errores } \\
\text { Espera por atención } \\
\text { Exactitud de las explicaciones } \\
\text { Horario de atención }\end{array}$ & $\begin{array}{l}\text { Al-Hawari, Hartley } \\
\text { and Ward [1] }\end{array}$ & $\begin{array}{l}\text { Banca telefónica } \\
\text { Accesos a sitios Web y la } \\
\text { banca de Internet }\end{array}$ \\
\hline $\begin{array}{l}\text { Sharma and Mehta } \\
\text { [52] }\end{array}$ & $\begin{array}{l}\text { Estructura física } \\
\text { Ambiente interno } \\
\text { Disposición de los recursos }\end{array}$ & Yavas [57] & $\begin{array}{l}\text { Elementos tangibles } \\
\text { Fiabilidad } \\
\text { Capacidad de respuesta } \\
\text { Seguridad } \\
\text { Empatía }\end{array}$ \\
\hline
\end{tabular}




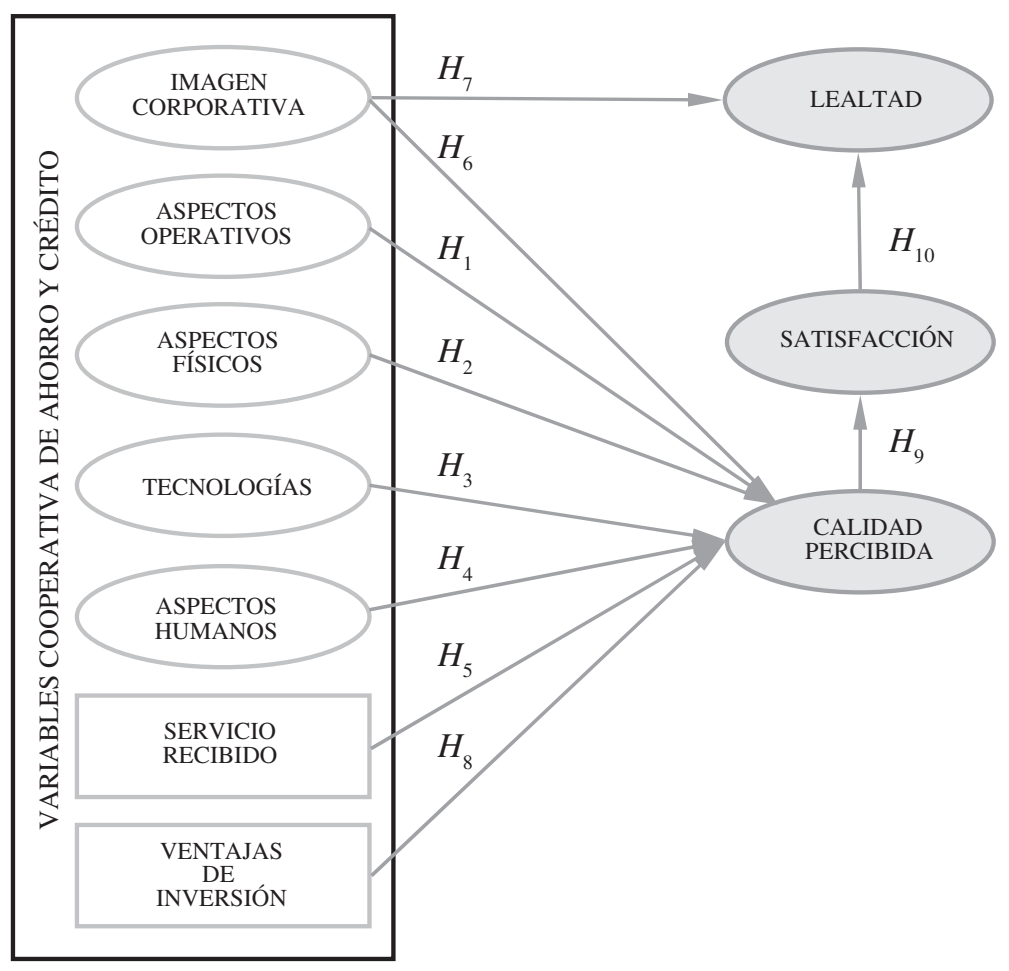

Figura 1. Modelo de relaciones.

Figura 1, que se encuentra basado en diez hipótesis, las que se justifican a continuación para el caso de estudio.

Los aspectos relacionados con el desempeño en la prestación del servicio de la cooperativa de ahorro y crédito, es decir, el tiempo de entrega, la puntualidad, la variedad de los servicios ofrecidos, la cantidad de errores cometidos por el personal, entre otros, corresponden a los aspectos operativos de cualquier organización. La relación de causalidad entre los aspectos operativos y la calidad percibida corresponde a la primera hipótesis de la investigación $[42,56]$.

H1: Los aspectos operativos de la cooperativa de ahorro y crédito influyen directamente en la calidad de servicio percibida.

Respecto de los aspectos físicos de la cooperativa de ahorro y crédito, es decir, lo que guarda relación con la apariencia de la estructura, el ambiente interno de la misma, la distribución de los recursos, entre otros aspectos, poseen una influencia directa sobre la calidad percibida $[11,20,49,52]$. Así, la segunda hipótesis de la investigación queda estructurada de la siguiente manera.

H2: Los aspectos físicos de la cooperativa de ahorro y crédito poseen un efecto directo y positivo en la calidad de servicio percibida.

Es necesario contar con innovaciones tecnológicas que busquen eliminar el trabajo repetitivo y manual, facilitando las transacciones. Dentro de estas innovaciones se encuentra la banca electrónica y la banca telefónica, las cuales influyen positivamente en la calidad percibida $[4,8,20,31]$. De esta manera se plantea la tercera hipótesis del trabajo.

H3: Las tecnologías que ofrece la cooperativa de ahorro y crédito afectan directamente a la calidad del servicio percibida.

Dentro de los aspectos humanos de toda organización se incluyen, entre otros elementos, todas las actitudes y capacidades que los empleados utilizan al momento de entregar el servicio. Por lo mismo, la cuarta hipótesis de la investigación plantea la existencia 
de una influencia positiva de los aspectos humanos en la calidad del servicio percibida $[4,20,56]$.

H4: Los aspectos humanos de la cooperativa de ahorro y crédito tienen un efecto directo y positivo sobre la calidad de servicio percibida.

El conjunto de actividades realizadas por la cooperativa de ahorro y crédito para responder a las necesidades de los clientes corresponde al servicio recibido por estos [7, 19, 49, 57]. Para identificar la relación entre el servicio recibido y la calidad percibida se procede a plantear la quinta hipótesis de la investigación.

H5: El servicio recibido por los clientes jóvenes de la cooperativa de ahorro y crédito influye directa y positivamente en la calidad del servicio percibida.

Según algunos autores [17, 34, 50-51], una imagen de empresa transparente afectaría positivamente la impresión que la población posee de la organización. La sexta hipótesis del trabajo se plantea para establecer una relación entre la imagen corporativa y la calidad de servicio percibida.

H6: La imagen corporativa de la cooperativa de ahorro y crédito posee un efecto directo y positivo en la calidad del servicio percibida.

Una imagen corporativa sólida es capaz de generar lealtad en los clientes, ya que, estos se sienten atraídos por asociarse con la imagen que la empresa o lo que sus productos representan [16]. Por lo tanto, los consumidores son más leales a aquellas empresas con la capacidad de proyectar una positiva imagen corporativa [15, 22, 34]. A continuación se presenta la séptima hipótesis, que propone una relación de causalidad entre la imagen corporativa y la lealtad.

H7: La imagen corporativa de la cooperativa de ahorro y crédito influye directa y positivamente en la lealtad de los clientes jóvenes.

Las ventajas de inversión de una empresa corresponden a todos los elementos económicos que hacen atractiva una determinada organización (beneficios de rentabilidad, solvencia financiera, tipos de préstamos ajustados a las necesidades de cada cliente, entre otros). Así, a continuación se plantea la hipótesis que establece la relación que existiría entre las ventajas de inversión y la calidad percibida [1, 45, 49].

H8: Las ventajas de inversión de la cooperativa de ahorro y crédito influyen directa y positivamente en la calidad del servicio percibida.

La satisfacción depende del grado en que se cubren las expectativas previas del cliente y del rendimiento percibido por este $[54,57]$. Un cliente que utiliza un servicio estará con una mayor disposición a volver a usarlo en el futuro y recomendarlo si su experiencia fue satisfactoria [20, 30, 35, 43].

La satisfacción de los clientes es un importante objetivo para las instituciones financieras y una forma de alcanzarla es mediante la calidad del servicio percibida. La calidad del servicio percibida es un antecedente de la satisfacción, por lo tanto, la excelencia en la calidad de atención es un factor clave para conseguir la satisfacción de los clientes [2-3, 13, 18, 26, 29, 36-37, 41, 43, 53, 56]. Así, la novena hipótesis de la investigación se plantea de la siguiente manera.

H9: La calidad del servicio percibida influye positiva y directamente en la satisfacción de los clientes jóvenes de la cooperativa de ahorro y crédito.

La lealtad es un tributo o reconocimiento de los clientes, es habitual que aquellos consumidores que sienten un compromiso con una determinada empresa, producto y/o servicio, manifiesten un proceso de recompra en el futuro, independientemente de las influencias situacionales y de las estrategias de marketing adoptadas por otras empresas para llamar su atención [39, 44, 48]. Por otro lado, la lealtad puede ser producto de diferentes motivos, por ejemplo, la inercia o la falta de alternativas [9]. Es necesario que las empresas reconozcan la importancia del valor añadido del servicio y de las relaciones con los clientes, para conseguir el objetivo básico de mantener la lealtad de estos [6].

En general en todos los servicios existe una fuerte relación entre la satisfacción de los clientes y la lealtad, la satisfacción es una variable que antecede a la lealtad [16, 23, 32, 44, 53, 57]. De este modo, se procede a plantear la hipótesis para la relación de causalidad entre la satisfacción de los clientes y su lealtad. 
H10: La satisfacción de los clientes jóvenes de la cooperativa de ahorro y crédito tiene un efecto positivo y directo en la lealtad de estos.

\section{METODOLOGÍA DE LA INVESTIGACIÓN EMPÍRICA}

Para validar las hipótesis anteriormente planteadas correspondientes a la base del modelo estructural, se recogió información de los clientes jóvenes de la cooperativa de ahorro y crédito bajo estudio, en un periodo comprendido entre el 26 de agosto y el 1 de septiembre de 2010. La población de la investigación corresponde a los clientes jóvenes de la cooperativa de ahorro y crédito, así, el tamaño muestral corresponde a 195 encuestas realizadas y válidas ${ }^{3}$ [38]. La elección del rango etario para los denominados "clientes jóvenes" se realiza en base a criterios planteados por la literatura, la que señala que el estrato comprendido de los 0-14 años corresponde a población infantil, de 15-29 años a población joven, de 30-44 años es adulto joven, de 45-65 años es adulto y de 65 o más años corresponde a adultos mayores [55]. No obstante en el presente estudio se agregarán los estratos joven y adulto joven, clasificándolos como "clientes jóvenes" a aquellos clientes con rango etario comprendido entre $25 \mathrm{y}$ 44 años, se omite realizar la encuesta a los clientes entre 15-25 años, ya que este grupo generalmente no posee ingresos, ni utiliza una gama importante de los productos ofrecidos por la cooperativa.

Para obtener la información necesaria para alcanzar los objetivos de la investigación y dar respuesta a las hipótesis planteadas, tras una revisión de la literatura especializada sobre el tema $[4,7-9,12-13,16,20,21$, $25-26,29,39,56]$, se diseña un cuestionario que ha sido sometido a un pretest que permite dar validez al mismo. Posteriormente se diseña el cuestionario definitivo que está compuesto por 25 preguntas (para conocer detalles del cuestionario contactar a los autores). A excepción de las características sociodemográficas, la información analizada fue medida a través de escalas de acuerdo con siete

3 Tamaño de la población: 34.126; Error absoluto admisible: $7 \%$; Probabilidad de que la muestra posea las mismas características de la población: 50\%; Probabilidad de que la muestra no posea las mismas características de la población: 50\%; Nivel de confianza: $95 \%$. puntos, desde 1 "Totalmente en Desacuerdo" hasta 7 "Totalmente de Acuerdo".

Así, la percepción de la calidad del servicio de los clientes jóvenes de la cooperativa de ahorro y crédito se estudia por medio de la metodología de ecuaciones estructurales, lo que nos permitirá confirmar la estructura teórica propuesta en la Figura 1, y dar respuesta a los objetivos e hipótesis planteados en esta investigación.

\section{ANÁLISIS DE RESULTADOS}

Al objeto de realizar un primer análisis de la opinión de los clientes sobre diferentes aspectos estudiados, tales como localización, imagen, amabilidad, servicio recibido, entre otras variables de interés, se realiza un análisis descriptivo exploratorio de los ítems planteados. En la Tabla 1 se muestran los valores medios y la desviación típica de cada uno de los ítems analizados.

De los resultados presentados en la Tabla 1 , se observa que todos los ítems analizados se sitúan por encima del punto medio de la escala de medición empleada. Este hecho pone de manifiesto, inicialmente, la existencia de opiniones positivas sobre los aspectos analizados.

Los ítems peor valorados se encuentran relacionados con el servicio de Internet, la espera en el servicio telefónico y la rapidez de atención, ítems que poseen valores inferiores a cinco.

En oposición a lo anterior, los ítems mejor evaluados corresponden a personal, amabilidad y voluntad, todos los cuales se relacionan con la variable latente aspectos operativos.

Una vez realizado el análisis descriptivo de los ítems materia de estudio, el objetivo a alcanzar se centra en la determinación de las dimensiones subyacentes que componen la variable calidad percibida por los clientes jóvenes de la cooperativa de ahorro y crédito.

Los resultados obtenidos del análisis factorial exploratorio (ver Tabla 2) muestran que los ítems se agrupan en cinco componentes que explican el $61,7 \%$ de la varianza total. 
Tabla 1. Estadísticos descriptivos de los ítems.

\begin{tabular}{|l|c|c|l|c|c|}
\hline \multicolumn{1}{|c|}{ Variables } & Media & $\begin{array}{c}\text { Desviación } \\
\text { típica }\end{array}$ & \multicolumn{1}{|c|}{ Variables } & Media & $\begin{array}{c}\text { Desviación } \\
\text { típica }\end{array}$ \\
\hline HORARIO & 6,10 & 1,304 & LENGUAJE & 6,33 & 1,078 \\
\hline AMPLITUD GAMA & 5,55 & 1,669 & SERVICIO RECIBIDO & 5,22 & 2,153 \\
\hline RAPIDEZ ATENCIÓN & 4,87 & 1,788 & CALIDAD SERVICIO & 6,07 & 1,208 \\
\hline RAPIDEZ SERVICIO & 5,51 & 1,718 & VENTAJAS & 5,30 & 1,725 \\
\hline LOCALIZACIÓN & 6,30 & 1,141 & RECOMENDACIÓN & 6,04 & 1,426 \\
\hline IMAGEN & 6,35 & 1,017 & TRANSPARENCIA & 6,03 & 1,403 \\
\hline VIGILANCIA & 6,31 & 1,010 & SATIS ATENCION & 6,03 & 1,262 \\
\hline EQUIPAMIENTO & 5,75 & 1,580 & SATIS CALIDAD & 6,06 & 1,263 \\
\hline ESPERA TELEFÓNICA & 4,94 & 1,348 & SATIS VARIEDAD & 5,76 & 1,453 \\
\hline SERVICIO WEB & 4,68 & 1,644 & INCENTIVO & 5,81 & 1,759 \\
\hline PERSONAL & 6,48 & 0,899 & UTILIZACIÓN FUTURA & 6,18 & 1,213 \\
\hline VOLUNTAD & 6,36 & 0,998 & PRIORIDAD FUTURA & 5,75 & 1,783 \\
\hline AMABILIDAD & 6,45 & 0,800 & & & \\
\hline
\end{tabular}

Tabla 2. Resultados del análisis factorial exploratorio.

\begin{tabular}{|l|c|c|c|c|r|}
\hline & C1 & C2 & C3 & C4 & C5 \\
\hline HORARIO & 0,595 & & & & \\
\hline AMPLITUD GAMA & 0,802 & & & & \\
\hline RAPIDEZ ATENCIÓN & 0,814 & & & & \\
\hline RAPIDEZ SERVICIO & 0,848 & & & & \\
\hline LOCALIZACIÓN & & 0,806 & & & \\
\hline IMAGEN & & 0,852 & & & \\
\hline VIGILANCIA & & 0,617 & & & \\
\hline EQUIPAMIENTO & & & 0,733 & & \\
\hline ESPERA TELEFÓNICA & & & 0,765 & & \\
\hline SERVICIO WEB & & & 0,617 & & \\
\hline PERSONAL & & & & 0,720 & \\
\hline VOLUNTAD & & & & 0,768 & \\
\hline AMABILIDAD & & & & 0,794 & \\
\hline LENGUAJE & & & & 0,743 & \\
\hline RECOMENDACIÓN & & & & & 0,908 \\
\hline TRANSPARENCIA & & & & & 0,908 \\
\hline Alfa de Cronbach & 0,772 & 0,638 & 0,670 & 0,743 & - \\
\hline Varianza explicada & 16,475 & 12,303 & 11,544 & 11,514 & 9,818 \\
\hline
\end{tabular}

Como se puede observar en la Tabla $2^{4}$, el primer componente explica un $16,5 \%$ de la varianza y posee cuatro ítems: horario, gama de servicios, rapidez

4 Para facilitar la comprensión de los resultados se han eliminado aquellos pesos menores a 0,4 por considerarse poco significativos dentro de los elementos [27]. de la atención y rapidez del servicio. El segundo componente se relaciona con los aspectos físicos, incluyendo la localización, imagen y vigilancia, este explica un $12,3 \%$ de la varianza. El tercer componente explica un $11,5 \%$ de la varianza y posee tres ítems que muestran los aspectos relacionados con las tecnologías y equipamiento, espera en los 
servicios telefónicos y el servicio Web. Para el cuarto componente se tiene que este explica un $11,5 \%$ de la varianza, y posee cuatro ítems que se relacionan con los aspectos humanos: personal, voluntad de ayudar, amabilidad del personal y lenguaje utilizado. Finalmente el quinto componente explica un $9,8 \%$ de la varianza, y se relaciona con la recomendación y la transparencia, en este caso no es posible justificar la fiabilidad mediante el coeficiente Alfa de Cronbach dado que sólo existen 2 ítems [27], sin embargo, se puede analizar la correlación entre las variables, obteniéndose que existe una correlación de 0,649 que es significativa a un nivel del $95 \%$.

Una vez determinada la existencia de cinco componentes, y aceptada la fiabilidad de las escalas subyacentes, se procede a la validación de las mismas, así como a la estimación del modelo propuesto previamente (ver Figura 1). Dicho análisis fue realizado a través de la aplicación de modelos de ecuaciones estructurales.

El modelo presentado en la Figura 1 establece que la variable latente endógena Calidad Percibida $\left(\eta_{1}\right)$ está causalmente relacionada con 7 variables latentes exógenas, Aspectos Operativos $\left(\xi_{1}\right)$, Aspectos Físicos $\left(\xi_{2}\right)$, Tecnologías $\left(\xi_{3}\right)$, Aspectos Humanos $\left(\xi_{4}\right)$ e Imagen Corporativa $\left(\xi_{5}\right)$, y las variables exógenas Servicio Recibido y Ventajas de Inversión, las cuales son indicadores directos representados por la notación $X_{6}$ y $X_{7}$ respectivamente. Los coeficientes $\gamma$ representan la relación entre cada elemento y la Calidad Percibida. La metodología asume la posibilidad de que todos los elementos no expliquen perfectamente la dimensión, para solucionar este inconveniente se incluye un término de perturbación en el constructo Calidad Percibida $\left(\zeta_{1}\right)$ [33].

En el caso del constructo Satisfacción $\left(\eta_{2}\right)$ solo se encuentra relacionado con la variable latente endógena Calidad Percibida $\left(\eta_{1}\right)$ mediante el coeficiente $\beta_{21}$, en este caso se incluye el término de perturbación $\zeta_{2}$.

La variable latente Lealtad $\left(\eta_{3}\right)$ está relacionalmente causada por dos constructos. Uno del tipo endógeno, Satisfacción $\left(\eta_{2}\right)$, y otro del tipo exógeno, Imagen Corporativa $\left(\xi_{5}\right)$, también se incluye el término de perturbación $\zeta_{3}$ con la misma intención que en el caso anterior.
A continuación se presentan las ecuaciones del modelo estructural siguiendo la notación expuesta:

$$
\begin{gathered}
\eta_{1}=\xi_{1} \mathrm{y}_{11}+\xi_{2} \mathrm{y}_{12}+\xi_{3} \mathrm{y}_{13}+\xi_{4} \mathrm{y}_{14}+\xi_{5} \mathrm{y}_{15} \\
+\mathrm{x}_{6} \mathrm{y}_{16}+\mathrm{x}_{7} \mathrm{y}_{17}+\zeta_{1} \\
\eta_{2}=\eta_{1} \beta_{21}+\zeta_{2} \\
\eta_{3}=\eta_{2} \beta_{32}+\xi_{5} \mathrm{y}_{35}+\zeta_{3}
\end{gathered}
$$

Se presentan los resultados del modelo propuesto mediante la realización de dos iteraciones, las cuales varían en cuanto a las relaciones causales del modelo original; para la estimación de los coeficientes de los modelos se utiliza el programa estadístico Amos 7.0.

En la Figura 2 se presentan los coeficientes determinados por el método de máxima verosimilitud, cada uno de ellos está asociado a un valor crítico que determina su significatividad estadística.

Para un nivel de confianza del 95\%, las relaciones que contengan un valor $\mathrm{p}$ mayor a 0,05 son no significativas estadísticamente. Por esta razón en la Figura 2 se identifican las relaciones no significativas, las que aparecen señaladas con una línea puntuada y sin el coeficiente estandarizado que la identifica.

En la Tabla 3 se muestran los índices de ajuste propios de la metodología para la primera iteración. La relación entre el Chi-Cuadrado y los grados de libertad del modelo indica un ajuste casi excelente [14]. Por otra parte, asumiendo que el GFI representa un índice análogo al $\mathrm{R}^{2}$, el valor del indicador es aceptable [33], sin embargo, respecto de las discrepancias relacionadas con la muestra (RMR) y a su aplicación en la población RMSEA, se esperan valores considerablemente menores para validar el modelo. Con respecto a los índices de ajuste incremental (NFI, TLI, IFI), no alcanzan valores deseables existiendo posibilidades de mejorar. Los valores de las medidas de ajuste parsimonial serán lógicos al momento de comparar dos modelos rivales.

Como una forma de evaluar el modelo de medida, también deben observarse los coeficientes que relacionan cada pregunta con las variables latentes. Las preguntas de AMPLITUDGAMA, 
EQUIPAMIENTO, ESPERATELEFONICA, SERVICIORECIBIDO y VENTAJAS poseen pesos considerablemente bajos, los cuales al ser eliminados generan una mejora del modelo, estos cambios se pueden observar detalladamente en la segunda iteración. Tras estimar el modelo con las modificaciones mencionadas se obtiene el diagrama producto de la segunda iteración (ver Figura 3).
Es importante observar las variaciones que este modelo presenta respecto del anterior. Así, se eliminan las preguntas que poseen pesos considerablemente bajos, con lo cual tecnologías ya no es un constructo, sino que solo una pregunta (SERVICIOWEB) que afecta directamente la variable endógena Calidad Percibida, además, se incorporan relaciones que mejoran los resultados de las hipótesis, se destaca

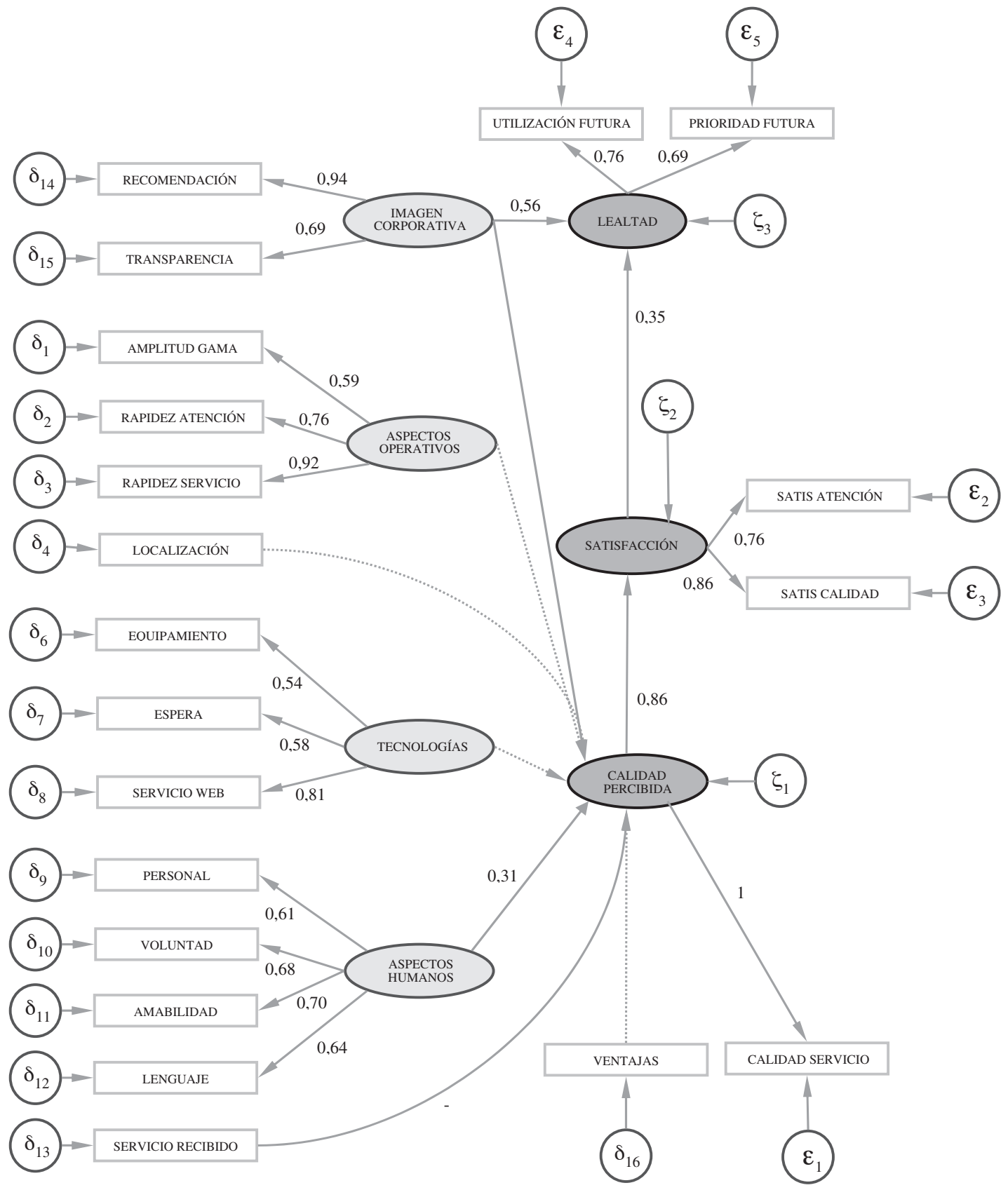

Figura 2. Modelo iteración 1. 
Tabla 3. Índice de ajuste primera iteración.

\begin{tabular}{|l|l|r|}
\hline \multicolumn{4}{|l|}{ ITERACIÓN 1 } & Chi-Cuadrado & 650,792 \\
\cline { 2 - 3 } & Grados de Libertad (G.L.) & 163 \\
\cline { 2 - 3 } Medidas de Ajuste & Chi-Cuadrado/G.L. & 3,993 \\
\cline { 2 - 3 } & P-Valor & 0,000 \\
\cline { 2 - 3 } & GFI & 0,729 \\
\cline { 2 - 3 } & RMR & 0,455 \\
\cline { 2 - 3 } & RMSEA & 0,124 \\
\hline \multirow{3}{*}{$\begin{array}{l}\text { Incremental } \\
\end{array}$} & NFI & 0,620 \\
\cline { 2 - 3 } & TLI & 0,626 \\
\cline { 2 - 3 } $\begin{array}{l}\text { Medidas de Ajuste } \\
\text { Parsimonial }\end{array}$ & IFI & 0,685 \\
\hline
\end{tabular}

que estas relaciones poseen sentido dentro del contexto de la organización estudiada. El diagrama obtenido mediante la "Iteración 2" se muestra a continuación en la Figura 3.

El siguiente paso es comparar los resultados obtenidos de ambas iteraciones, los cuales se muestran en la Tabla 4. En las medidas de ajuste global de la segunda iteración se observa que el valor de Chi-Cuadrado es muy inferior en la iteración 2 en comparación con la iteración 1, sin embargo, también se ha producido una disminución de los grados de libertad ocasionando que el componente ChiCuadrado / GL mejore en un 14,475\%. El indicador GFI aumenta considerablemente indicando que la variabilidad de los datos se ajusta aproximadamente en un $85 \%$ al modelo propuesto. Los valores de RMR y RMSEA también mejoran alcanzando mejores niveles que en la primera iteración. Con relación a las medidas de ajuste incremental se tiene que la variación del NFI produce que el modelo pase de un $62 \%$ a un $77,6 \%$. Los valores TLI e IFI se acercan más a la unidad mejorando cada uno más de un $17 \%$ [10].

En cuanto a los índices de ajuste de parsimonia, el PGFI mejora aproximadamente un $1 \%$, no obstante, no existen criterios para decidir cuando su valor es bueno o malo, simplemente se debe seleccionar el que posee mayor valor. Por el contrario para AIC, donde el que debe seleccionarse es el que muestra un menor valor [33], en este caso se tiene que el valor de AIC es inferior en la iteración 2, por lo tanto, esta mejora aproximadamente un $52 \%$ respecto de la iteración 1.

Finalmente la Tabla 4 indica que considerando todas las mejoras provocadas en la segunda iteración se ajusta en aproximadamente un 22,3\% más que la primera iteración.

Considerando que los resultados de esta estimación no arrojan correlación significativa entre las variables, no se realizan nuevas modificaciones de este modelo. Sin más modificaciones que realizar, se concluye que las hipótesis propuestas deben verificarse a partir de los estimadores obtenidos en la iteración 2 (ver Figura 3). En consecuencia, el no rechazo del modelo nos permite analizar la aceptación o no aceptación de cada una de las siete hipótesis planteadas.

Los resultados obtenidos, y presentados en la Figura 3, nos llevan a rechazar la primera hipótesis, es decir, no se ha comprobado la relación directa entre los aspectos operativos, compuestos por los ítems rapidez de la atención y la rapidez del servicio, y la calidad de servicio percibida por los clientes jóvenes de la cooperativa de ahorro y crédito.

Los resultados también llevan a rechazar la segunda hipótesis, que plantea que los aspectos físicos, compuesto por el ítem localización, que posee la cooperativa de ahorro y crédito influyen directa y 
Tabla 4. Comparación de los índices de ajuste primera iteración-segunda iteración.

\begin{tabular}{|c|c|c|c|c|}
\hline \multicolumn{2}{|c|}{ Índices de ajuste } & $\begin{array}{l}\text { Primera } \\
\text { iteración }\end{array}$ & $\begin{array}{l}\text { Segunda } \\
\text { iteración }\end{array}$ & $\begin{array}{l}\text { Porcentaje } \\
\text { mejoría }\end{array}$ \\
\hline \multirow{7}{*}{ Medidas de ajuste global } & Chi-Cuadrado & 650,792 & 276,601 & --- \\
\hline & $\begin{array}{l}\text { Grados de Libertad } \\
\text { (G.L.) }\end{array}$ & 163 & 81 & --- \\
\hline & Chi-Cuadrado/G.L. & 3,993 & 3,415 & 14,475 \\
\hline & P-Valor & 0,000 & 0,000 & --- \\
\hline & GFI & 0,729 & 0,849 & 14,134 \\
\hline & RMR & 0,455 & 0,218 & 52,088 \\
\hline & RMSEA & 0,124 & 0,112 & 9,677 \\
\hline \multirow{3}{*}{$\begin{array}{l}\text { Medidas de ajuste } \\
\text { ingremantal }\end{array}$} & NFI & 0,62 & 0,776 & 20,103 \\
\hline & TLI & 0,626 & 0,775 & 19,226 \\
\hline & IFI & 0,685 & 0,83 & 17,470 \\
\hline \multirow{2}{*}{$\begin{array}{l}\text { Medidas de ajuste } \\
\text { parsimonial }\end{array}$} & PGFI & 0,566 & 0,573 & 1,222 \\
\hline & AIC & 744,792 & 354,601 & 52,389 \\
\hline \multicolumn{4}{|c|}{ Porcentaje de mejoría general } & 22,309 \\
\hline
\end{tabular}

positivamente en la calidad de servicio percibida por los clientes jóvenes de la cooperativa.

Respecto de la tercera hipótesis planteada en el estudio, que establece que las tecnologías, compuesto por el ítem servicio Web, que ofrece la cooperativa de ahorro y crédito influyen directamente en la calidad de servicio percibida por los clientes jóvenes de la cooperativa, no ha sido posible ser comprobada.

Los resultados del estudio mostrados en la Figura 3 conducen a no rechazar la cuarta hipótesis planteada, que revela que los aspectos humanos de la cooperativa de ahorro y crédito, medida por los ítems personal, voluntad, amabilidad y lenguaje de los trabajadores influyen directa y positivamente en la calidad de servicio percibida por los clientes jóvenes de la cooperativa. Así, según los resultados obtenidos, se acepta la hipótesis planteada, por lo que una mejora en un punto de los aspectos operativos genera una variación en la calidad percibida de 0,23 puntos.

En relación con lo anterior, para los clientes jóvenes es relevante que el personal sea honesto y honrado para tener confianza en él, los resultados revelan una influencia de 0,60 puntos de la variable personal sobre el elemento aspectos humanos. Además, es necesario que el personal posea buena voluntad para ayudar cuando se le solicite, así, la variable voluntad ejerce una influencia de 0,68 puntos en los aspectos humanos. El ítem con mayor influencia sobre los aspectos humanos es amabilidad, con un efecto de 0,70 puntos, en este ítem se encuentran todos los tópicos relacionados con la cortesía por parte de los empleados al momento de prestar el servicio. Finalmente el aspecto lenguaje posee un efecto de 0,64 puntos sobre los aspectos humanos, ítem que considera la correcta utilización de lenguaje, en términos de la facilidad de comprensión y respeto.

Respecto de la quinta hipótesis que plantea que el servicio recibido por los clientes jóvenes de la cooperativa de ahorro y crédito influye directa y positivamente en la calidad del servicio percibida, es rechazada, ya que la variable servicio recibido ya había sido eliminada de la segunda iteración.

Para la sexta hipótesis de la investigación que establece que la imagen corporativa de la cooperativa de ahorro y crédito influye directa y positivamente en la calidad del servicio percibida por los clientes jóvenes de la cooperativa, es posible observar en los resultados obtenidos en la Figura 3, que para los clientes la imagen corporativa de la institución es un aspecto muy relevante, dentro de este ítem se encuentra la confianza que se genera producto de la transparencia de la imagen proyectada a la comunidad, y la eventual recomendación que se 
haría de la empresa a terceros. Dentro del constructo calidad percibida la imagen corporativa influye directamente, es decir, la percepción de calidad por parte de los clientes tendrá un incremento de 0,69 puntos al aumentar en una unidad la impresión de los clientes respecto de la imagen corporativa. Así, la sexta hipótesis es validada, siendo el ítem recomendación el que posee mayor efecto sobre la variable imagen corporativa.
La octava hipótesis que plantea que las ventajas de inversión de la cooperativa de ahorro y crédito influyen directa y positivamente en la calidad del servicio percibida por los clientes jóvenes de la cooperativa, es rechazada, por lo tanto, el elemento ventajas de inversión no resulta relevante a la hora de evaluar la calidad percibida de los clientes.

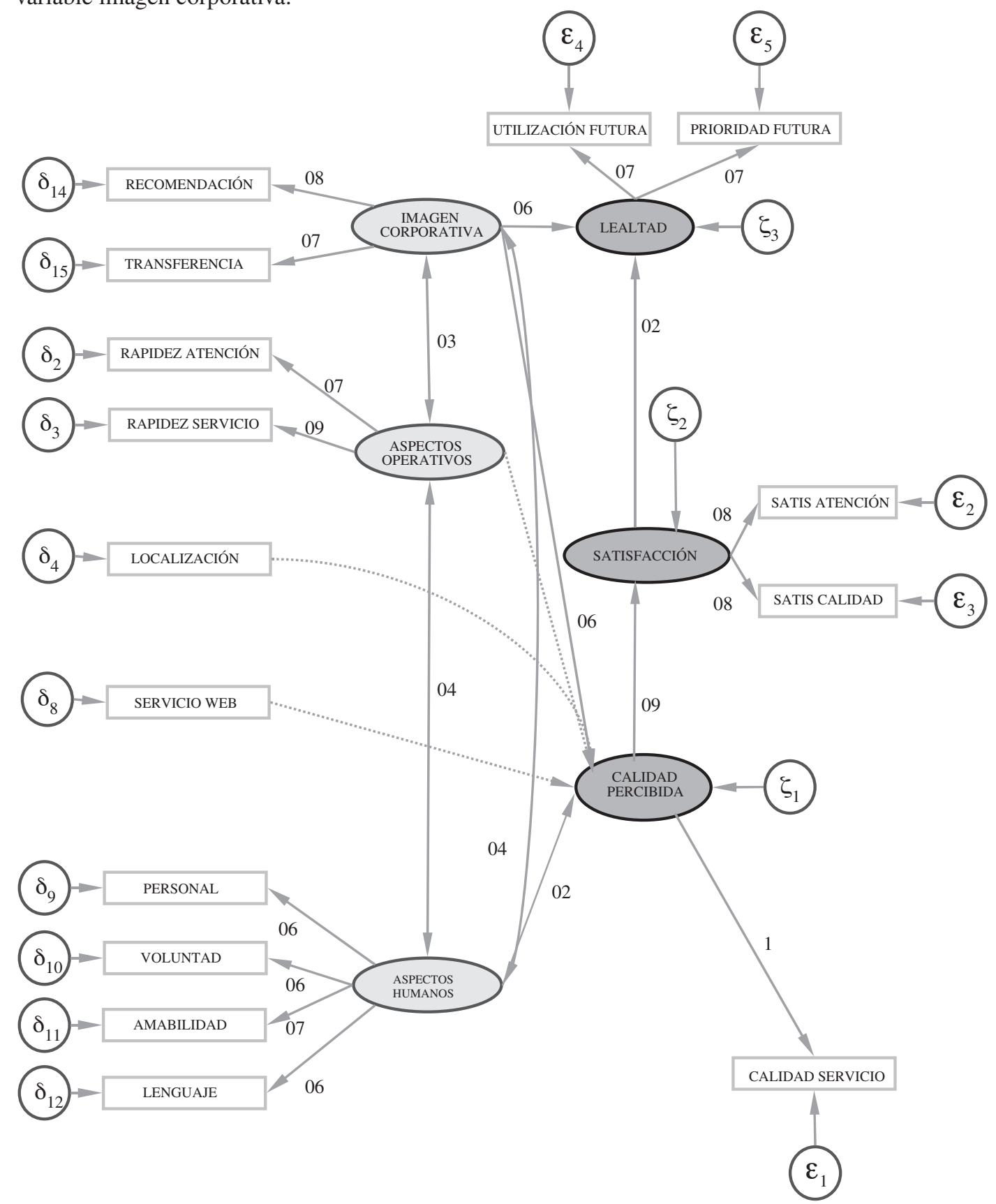

Figura 3. Modelo iteración 2. 
Respecto de la novena hipótesis planteada que señala que la calidad del servicio percibida por los clientes jóvenes de la cooperativa de ahorro y crédito influye positiva y directamente en la satisfacción de los clientes jóvenes, los resultados obtenidos en la Figura 3 conducen a no rechazar esta hipótesis. Así, una variación de la calidad percibida en un punto genera un aumento en la satisfacción de 0,9 puntos, es decir, si la empresa es capaz de crear valor y mejorar la percepción del cliente respecto de la calidad, entonces estará creando mayores posibilidades de que el consumidor cumpla o sobrepase sus expectativas.

Para los factores analizados en la investigación que afectan la lealtad de los clientes se tiene que respecto de la hipótesis diez planteada que señala que la satisfacción de los clientes jóvenes de la cooperativa de ahorro y crédito influye positiva y directamente en la lealtad de estos, los resultados obtenidos en la Figura 3 conducen a no rechazar esta hipótesis, es decir, existe una relación directa entre la satisfacción y la lealtad, la investigación muestra que al aumentar en un punto la satisfacción se produce un aumento en 0,23 puntos en la lealtad.

Ahora bien, respecto de la hipótesis siete que plantea que la imagen corporativa de la cooperativa de ahorro y crédito influye directa y positivamente en la lealtad de los clientes jóvenes, los resultados obtenidos en la Figura 3 conducen a no rechazar esta hipótesis. Así, la imagen corporativa de la institución posee una relación directa con la lealtad de los clientes, es decir, un aumento de la percepción de la imagen corporativa en una unidad provoca un aumento de la lealtad de 0,67 puntos, por lo tanto, los consumidores que tienen una percepción favorable acerca de la imagen tienen mayores posibilidades de ser leales a esa institución.

Finalmente y de acuerdo con los resultados obtenidos, la satisfacción es consecuencia de la calidad percibida, además la lealtad es consecuencia de la satisfacción del cliente, esto es congruente con los estudios presentados en la literatura.

\section{CONCLUSIONES Y FUTURAS INVESTIGACIONES}

Actualmente el sector financiero resulta atractivo para un gran número de competidores, las instituciones financieras chilenas se encuentran constantemente enfrentadas a grandes desafíos para capturar una cartera de clientes sólida. Todas las empresas que desean pertenecer o ingresar al sistema financiero deben establecer claramente la estrategia que utilizarán. La gran variedad de oferta en el mercado financiero, el crecimiento del poder adquisitivo de la clase media, la introducción de la mujer al campo laboral, la exigencia de servicios de mayor calidad y la presencia de consumidores más informados son algunos de los factores que las empresas deben manejar para lograr consolidarse, así se ha generado una preocupación por mejorar la calidad del servicio, buscando satisfacer las necesidades de los clientes para mantenerse vigente en el mercado.

En la presente investigación se planteó el caso de una cooperativa de ahorro y crédito, ubicada en la zona centro sur de Chile, en el estudio se ha presentado la importancia de conocer y adaptar nuevas metodologías de trabajo para conseguir comprender la forma de actuar de los consumidores, de este modo, las necesidades de la empresa de mantener clientes satisfechos y leales se responde con la creación de un modelo de ecuaciones estructurales basado en los componentes teóricos expuestos en la investigación.

El método de ecuaciones estructurales permite que el modelo incluya un análisis multivariante, al estudiar atributos complejos, como es el comportamiento del consumidor, por lo tanto, es una herramienta útil para ser aplicada a cualquier empresa del sector financiero, además, los modelos obtenidos son una buena aproximación de la realidad, ya que su base se sustenta en la teoría. El método de estimación utilizado corresponde al de máxima verosimilitud, este es el más representativo de las metodologías de ecuaciones estructurales y permite contrarrestar la significatividad de las relaciones propuestas.

Mediante la revisión del estado del arte se identifican siete factores que influyen en la calidad; sin embargo, solo dos de ellos poseen un efecto significativo en el constructo calidad percibida. El componente más influyente en la calidad percibida corresponde a la imagen corporativa, es decir, la imagen que la empresa ha logrado proyectar a los clientes, de este modo, la transparencia al momento de prestar sus servicios, ha generado en los clientes una sensación de confianza, además, la imagen positiva que proyecta la empresa 
produce que los clientes se motiven a recomendarla dentro de su círculos familiares y sociales. El segundo componente que ejerce influencia sobre la calidad percibida corresponde al factor aspectos humanos, es decir, los clientes requieren que se les trate amablemente, se les entregue un servicio cercano y de calidad. El factor más valorado dentro de los aspectos humanos corresponde a la amabilidad y cortesía mostrada por el personal, seguida de la voluntad de ayudar en la resolución de los conflictos, además, de un lenguaje apropiado, finalizando con un personal que destaque por la confianza generada por su honestidad y honradez, estos resultados proveen valor añadido de la investigación en lo que a la gestión de las entidad financiera se refiere.

La investigación no logra validar las relaciones establecidas entre los aspectos operativos, los aspectos físicos, las tecnologías, el servicio recibido y las ventajas de inversión sobre la calidad percibida; por lo tanto, no es posible asegurar que existe una influencia entre estos factores.

La satisfacción se encuentra en gran medida explicada por la calidad percibida, es decir, si los clientes sienten que la calidad del servicio es la adecuada dados sus requerimientos, consecuentemente se sentirán satisfechos con el servicio recibido.

En concordancia con la teoría, se corrobora que la lealtad está influenciada por la satisfacción de los clientes, además, se comprueba que existe una fuerte relación entre la imagen corporativa y la lealtad.

Esta investigación constituye los primeros pasos dentro de una línea más ambiciosa asociada a la medición de la calidad percibida en instituciones financieras, por lo que enfrenta una serie de limitaciones e investigaciones futuras. Así, se propone la posibilidad de repetir el estudio, considerando las mismas variables, para establecer la evolución del comportamiento de los clientes jóvenes de la cooperativa de ahorro y crédito en el tiempo, y de este modo generar un patrón que permita mejorar la toma de decisiones en el largo plazo logrando así actualizar bajo nuevos escenarios estos resultados. Del mismo modo sería interesante realizar un análisis que incorpore la relación entre la calidad percibida y variables del marketing sensorial (decoración, vestimenta del personal, entre otros). Finalmente, se debe considerar la realización de un análisis segmentado de la población, agrupando las respuestas, según género, edad, años de antigüedad como cliente, entre otros, identificando estrategias específicas para cada segmento.

\section{REFERENCIAS}

[1] M. Al-Hawari, N. Hartley and T. Ward. "Measuring banks' automated service quality: a confirmatory factor analysis approach". Marketing bulletin. Vol. 1, Issue 16, pp. 1-19. 2005.

[2] E. Anderson and M. Sullivan. "The antecedents and consequences of customer satisfaction for films". Marketing science. Vol. 12, Issue 2, pp. 125-143. 1993.

[3] E. Anderson, C. Fornell and D. Lehmann. "Customer satisfaction market share, and profitability: findings from Sweden". Journal of marketing. Vol. 58, Issue 3, pp. 53-66. 1994.

[4] J. Atenas. "Estrategias bancarias aplicables a las bibliotecas: la satisfacción y fidelización de usuarios". Revista de bibliotecnología y ciencias de la información. Vol. $7 \mathrm{~N}^{\mathrm{o}} 23$, pp. 1-12. 2006.

[5] A. Athanassopoulos. "Customer satisfaction cues to support market segmentation and explain switching behavior". Journal of business research. Vol. 47, Issue 3, pp. 191207. 2000.

[6] C. Barroso. "Marketing bancario, un enfoque estratégico". Esic Editorial. Edición 1, pp. 169-175. Madrid, España. 1995.

[7] M. Bath. "Correlates of service quality in Banks: an empirical investigation". Journal of services research. Vol. 5, Issue 1, pp. 77-99. 2005.

[8] H. Bauer, M. Hammerschmidt and T. Falk. "Measuring the quality of e-banking portals". International journal of bank marketing. Vol. 23, Issue 2, pp. 153-175. 2005.

[9] N. Bendapudi and L. Berry. "Customers' motivations for maintaining relationships with service providers". Journal of retailing. Vol. 73, Issue 1, pp. 15-37. 1997.

[10] P. Bentler and D. Bonett. "Significance test and goodness of fit in the analysis of covariance structures". Psychological bulletin. Vol. 88, Issue 3, pp. 588-606. 1980. 
[11] L. Berry. "Service marketing is different". Business. Vol. 30, Issue 1, pp. 24-29. 1980.

[12] C. Bhattacharya. "When customers are members: customer retention in paid membership context". Journal of the academy of marketing science. Vol. 26, Issue 1, pp. 31-44. 1998.

[13] J. Bigné, M. Moliner, T. Vallet y J. Sánchez. "Un estudio comparativo de los instrumentos de medición de la calidad de los servicios públicos". Revista española de investigación de marketing ESIC. Vol. 1 No 1, pp. 33-53. 1997.

[14] P. Brooke, D. Russell and J. Price. "Discriminant validation of measures of job satisfaction, job involvement, and organizational commitment". Journal of applied psychology. Vol. 73, Issue 2, pp. 139145. 1998.

[15] J. Cerviño. "La globalización de las marcas españolas: estrategia internacional e imagen de marca del Santander". Universia Business Review. Vol. 17 No especial, pp. 60-73. 2008.

[16] C. Churchill y S. Halpern. "Cómo desarrollar la lealtad del cliente". USAID's microenterprise best practices, pp. 5-57. 2001.

[17] J. Costa. "La imagen de marca: un fenómeno social”. Ediciones Paidós Ibérica S.A. México, pp. 25-27. 2004.

[18] J. Cronin and S. Taylor. "Measuring service quality: a reexamination and extension". Journal of marketing. Vol. 56, pp. 55-68. 1992.

[19] J. Dávila y M. Romero. "Estrategias de calidad del servicio en instituciones bancarias: factores que influyen en la calidad del servicio percibida". XII Jornadas hispanolusas de gestión científica, pp. 2821-2832. 2007.

[20] M. de la Cerda y M. Rubio. "Calidad en servicios en bancos, una estrategia en la captación de clientes". Universidad de San Carlos de Guatemala, pp. 11-36. 2006.

[21] H. de la Fuente, M. Marzo y M. Reyes. "Análisis de la satisfacción universitaria en la facultad de ingeniería de la Universidad de Talca". Ingeniare. Revista chilena de ingeniería. Vol. $18 \mathrm{~N}^{\circ}$ 3, pp. 350-363. 2010.

[22] C. Fandos y C. Flavián. "Influencia del valor de marca del jamón con do Teruel sobre la satisfacción y la intención de compra del consumidor". Departamento de economía y dirección de empresas. Universidad de
Zaragoza. Estudios sobre consumo. Vol. 77, pp. 77-87. 2006.

[23] C. Fornell. "A national customer satisfaction barometer: the Swedish experience". Journal of marketing. Vol. 56, pp. 6-21. 1992.

[24] J. Ganesh, M. Arnold and K. Reynolds. "Understanding the customer base of service providers: an examination of the difference between switchers and stayers". Journal of marketing. Vol. 64, Issue 3, pp. 65-87. 2000.

[25] A. González. "El reto de la calidad del servicio financiero". Investigaciones europeas de dirección y economía de la empresa. Vol. 6 $\mathrm{N}^{\circ}$ 1, pp. 71-92. 2000.

[26] M. Goode, L. Moutinho and C. Chien. "Structural equation modeling of overall satisfaction and full use of services for ATMs". International Journal of Bank Marketing. Vol. 7, pp. 4-11. 1996.

[27] J.F. Hair, R.E. Anderson, R.L. Tatham y W.C. Black. "Análisis multivariante". Prentice Hall. Madrid, España. 1999.

[28] K. Ishikawa. "¿Qué es el control de calidad? La modalidad Japonesa”. Grupo Editorial Norma, pp. 55-58. 1986.

[29] A. Jamal and K. Nasser. "Customer satisfaction and retail banking: an assessment of some of the key antecedents of customer satisfaction in retail banking". International Journal of Bank Marketing. Vol. 20, Issue 4, pp. 146160. 2002.

[30] R. Jayanti and A. Jackson. "Service satisfaction: an exploratory investigation of three models". Journal Article. Vol. 18, pp. 603-609. 1991.

[31] C. Jayawardhena. "Measurement of service quality in internet banking: the development of an instrument". Journal of Marketing Management. Vol. 20, Issue 1, pp. 185-208. 2004.

[32] T. Jones and W. Sasser. "Why satisfied customers defect". Harvard Business Review. Vol. 73, Issue 6, pp. 88-99. 1995.

[33] K. Jöreskog and D. Sörbom. "Lisrel 8: User's reference guide". Scientific Software International. Segunda edición, pp. 310-320. USA. 1996.

[34] K. Karounga y M. Herreros. "La imagen corporativa de las entidades financieras: el caso del Banco Sabadell en Cataluña". 
Universidad Autónoma de Barcelona, pp. 27212. 2005.

[35] P. Kotler. "Dirección de marketing". Pearson Prentice Hall. Edición del milenio, pp. 218252. USA. 2000.

[36] W. Lassar, C. Manolis and R. Windsor. "Service quality perspectives and satisfaction in private banking". Journal of services marketing. Vol. 14, Issue 3, pp. 244-271. 2000.

[37] F. Lloréns. "Medición de la calidad del servicio: una aproximación a diferentes alternativas". Universidad de Granada. Granada, España. 1996.

[38] C. Martínez. "Estadística Comercial". Grupo Editorial Norma Educativa. Colombia, pp. 223-230. 1994.

[39] J. Marval. "Diseño de un modelo de investigación de mercados para la formulación de programas de fidelización en la categoría de banca minorista". Revista científica de UCES. Vol. 14 Nº 1, pp. 78-99. 2010.

[40] J. Miranda, P. Rey y R. Weber. "Predicción de fugas de clientes para una institución financiera mediante support vector machines". Revista de Ingeniería de Sistemas. Vol. 19, pp. 49-66. 2005.

[41] V. Morales y A. Hernández. "Calidad y satisfacción en los servicios: conceptualización”. Revista Digital, Año $10 \mathrm{~N}^{\circ} 73$. Junio de 2004. Fecha de consulta: Noviembre de 2010. URL: www.efdeportes. com.

[42] T. Newby and S. McManus. "Customer service pocketbook". Management pocketbooks Ltd. Segunda edición, pp. 1-21. 2002.

[43] R. Oliver. "A cognitive model of the antecedents and consequences of satisfaction decision". Journal of Marketing Research. Vol. 17, pp. 460- 469. 1980.

[44] R. Oliver. "Whence consumer loyalty?" Journal of Marketing. Vol. 63, pp. 33-44. 1996.

[45] A. Paswan, N. Spears, R. Hasty and G. Ganesh. "Search quality in the financial services industry: a contingency perspective". Journal of Services Marketing. Vol. 18, Issue 5, pp. 324-339. 2004.

[46] M. Paulin, J. Perrien, R. Ferguson, A. Álvarez and L. Seruya. "Relational norms and client retention: external effectiveness of commercial banking in Canada and México".
International Journal of Bank Marketing. Vol. 16, Issue 1, pp. 24-31. 1998.

[47] E. Ramusson. "Complaints can build relationships. Sales and marketing management”. Vol. 151, Issue 9, pp.89-90. 1999.

[48] F. Reichheld. "El efecto de la lealtad: la fuerza detrás del crecimiento, la rentabilidad y la creación de valor en las empresas". Grupo Editorial Norma, pp. 193-201. 1996.

[49] S. Rodríguez y J. Rodríguez. "Calidad de servicio: Exigencia actual para entidades financieras competitivas", pp. 93-107. 1994.

[50] J. Sánchez y J. Cubillos. "Imagen-Ciudad y calidad percibida de los servicios". Revista de cultura y ciencias sociales". Vol. 45, pp. 79-87. 2005.

[51] J. Sánchez, S. del Barrio y F. Fuentes. "Los atributos calidad y marca-denominación de origen en el proceso de decisión del consumidor granadino". Universidad de Granada, pp. 30-47. Granada, España. 1996.

[52] A. Sharma and V. Mehta. "Service quality perceptions in financial services - a case study of banking services". Journal of Services Research. Vol. 4, Issue 2, pp. 205-223. 2004

[53] S. Taylor and T. Baker. "An assessment of the relationship between service quality and customer satisfaction in the formation of consumers' purchase intentions". Journal of Retailing. Vol. 70, Issue 2, pp. 163-178. 1994.

[54] D. Tse and P. Wilton. "Models of consumer satisfaction formation: an extension". Journal of Marketing Research. Vol. 25, pp. 204-212. 1998.

[55] G. Valdivia. "Aspectos de la situación de salud del adulto y senescente en Chile". Boletín Escuela Medicina Pontificia Universidad Católica de Chile. Vol. 23, pp. 18-22. 2004.

[56] L. Valdunciel, M. Flórez y J. Dávila. "Análisis de la calidad del servicio que prestan las entidades bancarias y su repercusión en la satisfacción del cliente y la lealtad hacia la entidad". Revista Asturiana de Economía. Vol. $1 \mathrm{~N}^{\circ}$ 38, pp. 79-99. 2007.

[57] U. Yavas, Z. Bilgin and D. Shemwell. "Service quality in the banking sector in an emerging economy: a consumer survey". International Journal of Bank Marketing. Vol. 15, Issue 6, pp. 217-223. 1997. 